\title{
Produktion von Vorlesungsvideos mit Greenscreen-Technik
}

\author{
M. Beitelschmidt*, D. Bernstein, J. Bieber, M. Schuster
}

Professur für Dynamik und Mechanismentechnik, Institut für Festkörpermechanik, Fakultät Maschinenwesen, TU-Dresden

\begin{abstract}
Sollen Vorlesungsvideos zum Download oder als Livestream mit guter Qualität produziert werden, ist die Einrichtung eines Videostudios unumgänglich. Der Aufbau eines Studios mit einfachen Mitteln in einem umfunktionierten Seminarraum wird beschrieben. Dabei kommt Greenscreen-Technik zum Einsatz. Diese ermöglicht es, den Sprecher oder die Sprecherin vor seine digital generierten Inhalte zu stellen und inn wie vor einer klassischen Tafel agieren zu lassen. Erfahrungen bei der Einrichtung und dem Betrieb des Studios werden dargestellt sowie eine Bilanz nach mittlerweile ca. 100 produzierten Lehrstunden gezogen.
\end{abstract}

If lecture videos are to be produced for download or as livestream with good quality, the establishment of a video studio is inevitable. The set-up of a simple studio in a converted seminar room is described. Green screen technology is used. This makes it possible to place the speaker in front of his digitally generated content and let him act as if he was in front of a classical blackboard. Experiences with the installation and operation of the studio are presented and conclusions are drawn after approximately 100 hours produced lessons. 


\section{Einleitung und Rückblick}

Im Rahmen der Online-Lehre werden von den Mitarbeiterinnen und Mitarbeitern der Professur für Dynamik und Mechanismentechnik seit dem Sommersemester 2020 Lehrvideos für Vorlesungen, Vorrechenübungen und ähnliche Formate produziert. Die Dozentinnen und Dozenten der Professur setzten dabei von Anfang an darauf, gewohnte, bereits seit Jahren digitale, Vortragsformate in eine Videodarstellung umzusetzen. Lehrveranstaltungen der Professur setzen sich häufig aus einem Mix aus "live" handgeschriebenen Anteilen auf Basis von Microsoft OneNote auf Tablet-PC sowie PowerPoint Folien, ggf. mit handschriftlichen Ergänzungen, zusammen. Diese Präsentationsform wurde, speziell im Sommersemester 2020, mit Hilfe des Video-Produktionsprogramms Open Broadcaster Software ${ }^{\circledR}$ (OBS), teilweise mit Hineinschneiden eines kleinen Dozentenvideos (Abb. 1) umgesetzt.

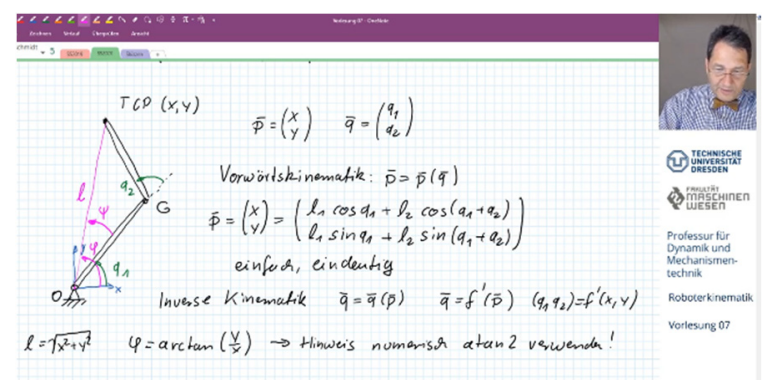

Abb. 1: Screenshot eines konventionellen Lehrvideos mit kleinem Dozentenbild

Im Sommersemester 2020 wurden die Videos von den Dozenten in der Regel im Homeoffice hergestellt. Das hat zwar, auch angesichts der kurzen Vorbereitungszeit, gut funktioniert, hat aber aus Sicht der Autoren folgende Nachteile:

- Die Qualität heimischer Kameras, z.B. einer Laptop-Kamera, ist nicht optimal. Im Sommer 2020 war es zudem quasi unmöglich, Kameras zu beschaffen.

- Hochwertige Tonqualität benötigt gute Mikrofone. Auch diese waren entweder nicht vorhanden oder nicht beschaffbar.

- Es war den Dozenten nicht möglich, im heimischen Umfeld einen Aufbau herzustellen, bei dem sie die Videos in der besseren
Sprechhaltung im Stehen produzieren konnten.

- Bei der Produktion aufwändiger Szenarien, z.B. mit Einbindung eines Experiments, fehlte eine Aufnahmeassistenz.

Um die genannten Nachteile auszuräumen, beschlossen wir, in den Räumlichkeiten der Professur ein Studio einzurichten, in dem die Vorlesungsvideos in professionellerer Umgebung produziert werden können. Dazu wurde ein Seminarraum, der früher für bis zu 30 Studierende geeignet war, umgerüstet. Dort können die Dozenten und Dozentinnen der Professur entweder Videos aufzeichnen, die später zum Download angeboten werden, oder Beiträge, die über Streamingportale wie YouTube verbreitet werden, produzieren. Von beiden Formaten wurde ab dem Wintersemester 2020 umfangreich Gebrauch gemacht.

\section{Greenscreen Technik}

Die Autoren sind davon überzeugt, dass ein Vorlesungsvideo massiv an Qualität gewinnt, wenn die sprechende Person im Video zu sehen ist. Das hat folgende Gründe:

- Die Sprachverständlichkeit steigt, wenn die Mimik und die Mundbewegungen der sprechenden Person sichtbar sind. Das ist gerade im Hinblick auf nicht-muttersprachliche Hörerinnen und Hörer sowie für englischsprachige Vorlesungen der Professur besonders wichtig.

- Die sprechende Person kann ihre Ausführungen durch Gesten untermalen. Zudem ist es möglich, Demonstrationen und Experimente einzubinden.

- Die Verbindlichkeit eines Vortrags steigt, wenn ein sprechender Mensch sichtbar ist und nicht nur Ton aus dem "Off" kommt.

Moderne Fernsehstudios sind heute in der Regel Greenscreen-Studios. Das Studio ist weitgehend leer und mit einer grünen Rückwand oder sogar einem grünen Fußboden ausgestattet. Mit dieser Technik ist es möglich, die sprechende Person im Vordergrund aufzunehmen, "freizustellen" und im Hintergrund einen beliebigen Inhalt einzublenden. 
Die Greenscreen-Technik als eine Form des "Chroma Keying" basiert auf digitaler Bildbearbeitung. Dabei erkennt die Video-Software im Vordergrundvideo die möglichst einheitliche Farbe Grün im Hintergrund und schaltet diese Flächen auf transparent. Dieses Bild kann nun digital vor ein weiteres beliebiges statisches oder bewegtes Videobild gelegt werden.

Diese Technik sollte auch für die Videos der Professur für Dynamik und Mechanismentechnik zum Einsatz kommen. Damit ist es möglich, dass die präsentierende Person im Vordergrund (Abb. 4), vor ihren im Hintergrund eingeblendeten Inhalten wie vor der klassischen Tafel agieren kann. So kann z.B. mit der Hand auf virtuelle Inhalte im Hintergrund gezeigt werden (Abb. 2).

Damit versprechen sich die Autoren eine besonders lebendige Gestaltung der Videos. Diese Technik wird im Fernsehen unter anderem bei der Wettervorhersage eingesetzt, wenn die Meteorologinnen oder Meteorologen vor einer nur virtuell eingeblendeten Wetterkarte präsentieren.

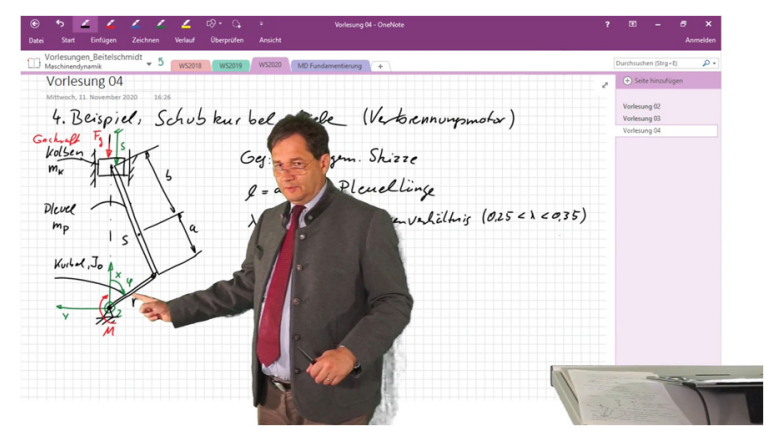

Abb. 2: Mögliches Agieren des Dozenten vor dem Hintergrundbild. Am unteren Rückenbereich ist ein "Schatten" erkennbar, der durch nicht optimale Ausleuchtung entsteht.

\section{Grundriss und Anordnung im Studio}

Wie bereits erwähnt, ist ein grüner Hintergrund erforderlich. Für das Format wurde entschieden, dass nur der Oberkörper der Lehrperson sichtbar sein soll, da das für eine universitäre Lehrveranstaltung ausreichend ist. Somit ist nur eine hängende, grüne Rückwand von Decke bis Boden erforderlich. Entspre- chende Tücher sind im Fachhandel für Videotechnik erhältlich.

Für ein gutes Ergebnis ist es erforderlich, dass der grüne Hintergrund möglichst einheitlich ausgeleuchtet ist. Dazu sind mehrere Scheinwerfer erforderlich, da bereits der Schattenwurf der präsentierenden Person den Grünton des Hintergrundes so verfälschen kann, dass die Video-Software diesen nicht mehr eindeutig als "Hintergrund" erkennt (Abb. 2).

Für ein gutes Agieren vor der Kamera und Zeigeoperationen auf dem virtuellen Inhalt ist es sehr wichtig, dass sich die sprechende Person vor dem bereits fertig abgemischten Gesamtbild sehen kann. Dazu wurde ein 65-Zoll Fernseher direkt gegenüber der Bewegungszone als Kontrollbildschirm aufgestellt.

Als beste Kameraposition hat sich eine Montage oben in der Mitte des Kontrollbildschirms herausgestellt. Das kommt der Funktion eines Teleprompters nahe, da die sprechende Person, wenn sie auf den Kontrollbildschirm schaut, quasi automatisch auch in die Kamera blickt.

Der Sprecherplatz mit einem schreibfähigen Tablet auf einem Pult wurde seitlich angeordnet. Das hat den Sinn, dass die präsentierende Person in der Regel seitlich am Rand des Bildes steht, wenn sie auf dem Tablet mit dem Stift Inhalte entwickelt, und damit nicht die virtuelle Tafel verdeckt. Für Zeigevorgänge und Interaktion mit dem Bild kann die Person das Pult verlassen und vor das virtuelle Bild treten (Bewegungsfläche in Abb. 3).

Für die Tonaufzeichnung wurde wahlweise sowohl das RODE-NT-USB-Mikrofon auf einem Stativ als auch ein Ansteck-Funkmikrofon von Sennheiser EW 100 G3 verwendet. Hinter dem Bildschirm, in angemessener Entfernung, ist der Operatorplatz angeordnet. Hier kann ein Aufnahmeassistent oder -assistentin Platz nehmen, um den Aufnahmevorgang zu überwachen, zwischen Aufnahmemodi zu wechseln, den Sprecher oder die Sprecherin auf inhaltliche und technische Pannen aufmerksam zu machen sowie bei Livestreams die Kommunikation mit den Hörerinnen und Hörern zu koordinieren. Die Anordnung des Studios ist in Abb. 3 schematisch dargestellt. 


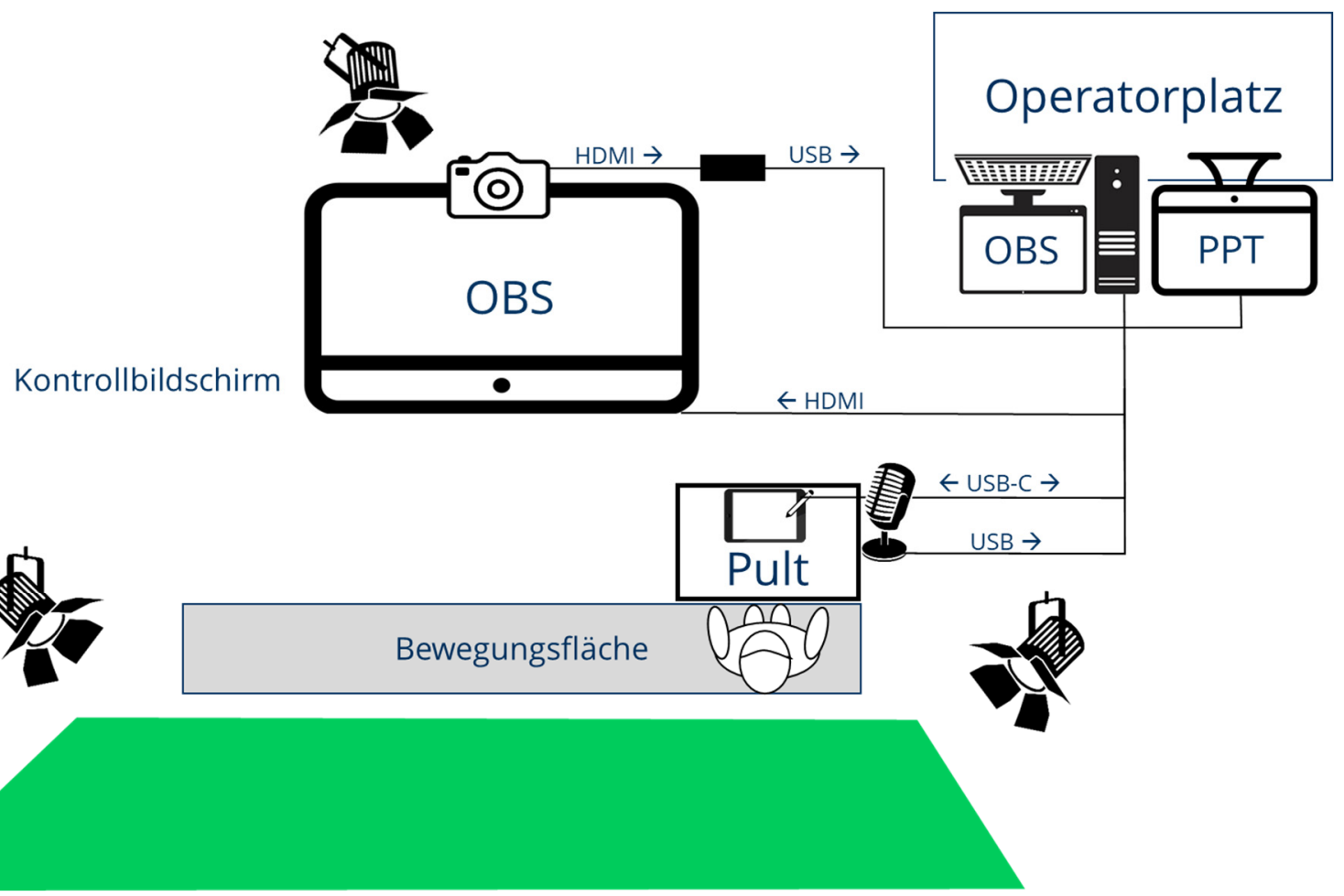

Abb. 3: Grundriss des Studios

Abweichend zur Symboldarstellung in Abb. 3 werden keine klassischen Scheinwerfer, sondern moderne LED Panels eingesetzt. Die Anordnung ist jedoch korrekt dargestellt: Eine Hauptlichtquelle direkt über dem Kontrollbildschirm leuchtet die sprechende Person aus. Durch die Breite von $30 \mathrm{~cm}$ dieses Panels wird ein scharfer Schattenriss der Person bereits teilweise abgemildert. Die beiden seitlichen Scheinwerfer beleuchten schräg nur die grüne Wand hinter der sprechenden Person, um den Schattenwurf weiter zu verringern.

Abb. 4 zeigt das Innere des Studios mit einem Sprecher. Oberhalb des Kontrollbildschirms ist die Kamera sowie die Hauptlichtquelle zu erkennen. Die eine der beiden seitlichen Lichtquellen (kleines weißes Rechteck im roten Kreis) ist für das Foto ausgeschaltet worden, um Gegenlicht im Bild zu vermeiden. Deswegen ist der Schatten der Person auf der grünen Wand relativ scharf erkennbar. Auf dem Kontrollbildschirm sieht der Sprecher das fertig zusammengesetzte Videobild.

Abb. 5 zeigt die Perspektive der Vortragenden im Studio. Vor dem Sprecher oder der Sprecherin liegt das Tablet auf dem Pult, dahinter ist das Mikrofon auf einem Stativ montiert. Ge-

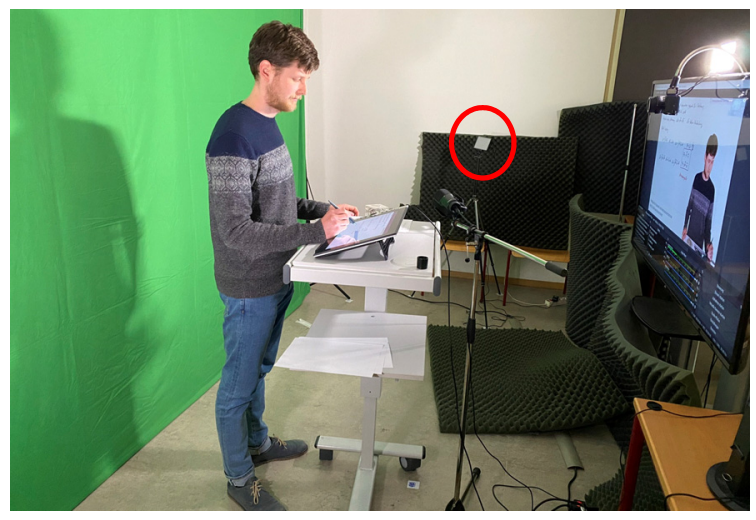

Abb. 4: Bild aus dem Studio (Foto: M. Beitelschmidt)

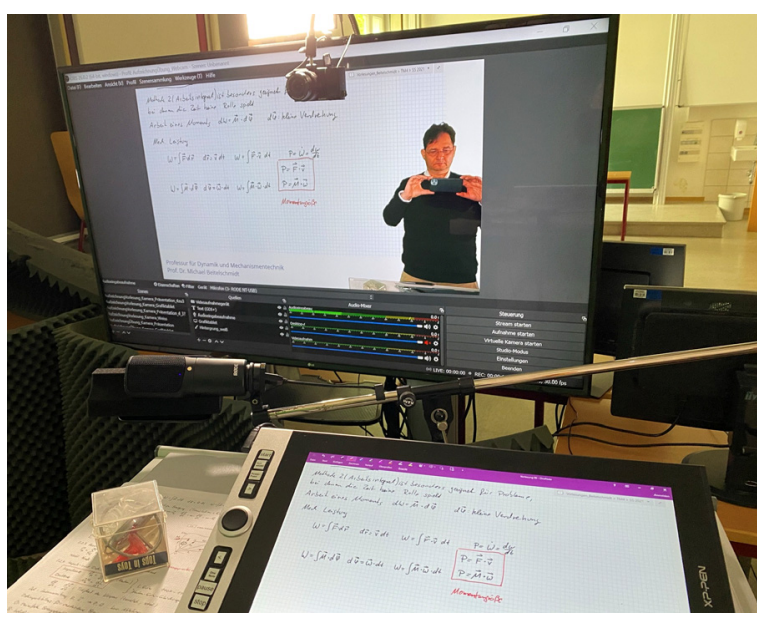

Abb. 5: Perspektive der Vortragenden (Foto: M. Beitelschmidt) 
genüber befindet sich der Kontrollbildschirm, auf dem sich die Vortragenden wie im Spiegel sehen.

\section{Software und Hardware}

Die gesamte Aufnahmetechnik wird von einem Standard-PC gesteuert, der von der Aufnahmeassistenz bedient wird. An diesem PC sind das jeweilige Mikrofon und die Kamera über einen HDMI-USB Konverter angeschlossen. Das stiftsensitive Tablet (XP-Pen Innovator 16) auf dem Sprecherpult wird mit USB-C über einen Adapter an einen DisplayPort des PC angeschlossen (Abb. 3). Die auf dem Rechner genutzte Software OBS beherrscht Greenscreen. Der PC ist mit einem Intel Xeon E6-2667 Prozessor $(3,2 \mathrm{GHz})$ ausgestattet und verfügt über 32 GB Arbeitsspeicher. Die Leistungsfähigkeit des Geräts wird allerdings während der Videoaufnahme nicht ausgeschöpft. Am PC sind vier Bildschirme angeschlossen:

1. Auf einem Bildschirm sieht die Assistenz die OBS-Oberfläche.

2. Als zweiter Bildschirm fungiert der Kontrollbildschirm (Großbildfernseher) der Vortragenden, auf den der Assistenzbildschirm mit der OBS-Oberfläche dupliziert wird.

3. Auf einem dritten Bildschirm werden eingebundene PowerPoint-Präsentationen angezeigt.

4. Das Tablet am Sprecherpult ist ein weiterer Bildschirm.

Der Betrieb der vier Bildschirme ist mit den am PC vorhandenen Video-Ausgängen, einem DVIAusgang und zwei DisplayPorts, möglich. Die Schirme 1 und 2 erhalten über einen DisplayPort das gleiche Bildsignal, das durch einen externen Splitter dupliziert wird. Bildschirm 3 ist am DVI-Ausgang angeschlossen. Das Tablet als Bildschirm 4 ist mit einem speziellen Adapter mit dem zweiten DisplayPort verbunden.

Wie im ersten Abschnitt erwähnt, kommen vor allem zwei Darstellungsmodi zum Einsatz: Im OneNote-Modus sehen die Vortragenden ihre OneNote Seite auf dem Tablet und können darin mit dem Tablet-Stift schreiben. OBS greift diesen Screen ab und macht daraus den Bildschirmhintergrund, vor den das freigestellte Sprechervideo gelegt wird.

Im PowerPoint-Modus wird mit der Referentenansicht gearbeitet, auf dem dritten Bildschirm entsteht die Präsentationsansicht, die von OBS abgegriffen wird. Diese wird dann wieder mit dem Sprechervideo überlagert.

Mit Hilfe vorbelegter Funktionstasten auf dem Tablet (Abb. 5, linker Rand des Tablets) kann der Sprecher oder die Sprecherin auch selbständig zwischen den Modi umschalten sowie die Aufzeichnung starten, stoppen und pausieren. Bei einfachen Aufzeichnungen kann damit sogar auf die Assistenz verzichtet werden. Die Pausenfunktion können die Vortragenden z.B. nutzen, um Textpassagen oder Abbildungen in OneNote "offline" vorzubereiten und anschließend bei laufendem Video zu erläutern, was die Lebendigkeit erhöht.

Tabelle 1 zeigt eine ungefähre Aufstellung der Anschaffungskosten zur Einrichtung des Studios. Hinzu kommt der beschriebene PC, HDMI- und USB-Kabel sowie zwei 24"-Bildschirme. Stative für Mikrofon und Scheinwerfer waren an der Professur vorhanden.

Tabelle 1: Kostenaufstellung der wichtigsten Hardwarekomponenten

\begin{tabular}{|c|c|c|}
\hline Gerät & Typ & Preis \\
\hline Kamera & Sony Alpha 6000 & $500 €$ \\
\hline \multirow[t]{2}{*}{ Mikrofone } & $R O D E-N T$ & $150 €^{*}$ \\
\hline & $\begin{array}{l}\text { Sennheiser EW } 100 \\
\text { G3 (mit tragbarem } \\
\text { Sender und Empfän- } \\
\text { ger) }\end{array}$ & $700 €^{*}$ \\
\hline Tablet & XP-Pen Innovator 16 & $500 €$ \\
\hline $\begin{array}{l}65-\text {-oll } \\
\text { Bild- } \\
\text { schirm }\end{array}$ & LG 65UK6400 & $700 €^{*}$ \\
\hline $\begin{array}{l}\text { Beleuch- } \\
\text { tung }\end{array}$ & $\begin{array}{l}\text { Walimex Pro Nova } \\
300 \text { bzw. } 150\end{array}$ & $160 €$ \\
\hline $\begin{array}{l}\text { Hinter- } \\
\text { grund }\end{array}$ & $\begin{array}{l}\text { Neewer 2,6 } 63 \mathrm{~m} \\
\text { Greenscreen }\end{array}$ & $110 €$ \\
\hline
\end{tabular}

*musste nicht beschafft werden 


\section{Erfahrungen}

Prinzipiell gelang es schnell, im Studio gute Videos zu produzieren. Eine erste Lernschleife betraf die Kamera: Eine normale "Webcam", reicht für ein gutes Abtrennen des grünen Hintergrundes nicht aus. Auch wenn diese ein hochaufgelöstes Bild liefert, sorgt ein starkes Rauschen für Probleme beim Freistellen. Als sehr geeignet stellte sich eine Sony Alpha 6000 Kamera mit Full HD-Videoauflösung heraus.

Bei den Mikrofonen konnte noch kein grundsätzliches Optimum gefunden werden. Für Vortragende, die vor allem am Pult stehen und wenig vor dem Hintergrund agieren, liefert das stationäre Mikrofon am Stativ vor dem Pult den besseren Ton. Bewegt sich die Sprecherin oder der Sprecher viel vom Pult weg, ist das ansteckbare Funkmikrofon wahrscheinlich die bessere Wahl.

Nach der Produktion der ersten Vorlesungsserien fiel auf, dass, unabhängig von der Wahl des Mikrofons, zu viel "Hall“ im Ton zu hören war. Das ist sicher darauf zurückzuführen, dass der zum Studio umfunktionierte Seminarraum im Wesentlichen sechs schallharte und damit reflektierende Wand-, Boden- und Deckenflächen aufweist.

Deswegen wurde begonnen, schallabsorbierendes Material im Studio anzubringen (teilweise sichtbar auf Abb. 4 und Abb. 5). Hierbei wurde und wird bisher noch experimentell vorgegangen. Eine Messung z.B. der Nachhallzeit bei verschiedenen Anordnungen der Absorptionsmaterialien wurde noch nicht durchgeführt. Eine akustische Optimierung des Studios ist weiterhin noch in Arbeit.

Eine große Herausforderung für die Vortragenden ist die Interaktion mit inrem Vorschaubild auf dem Kontrollbildschirm. Nur so können sie z.B. auf Inhalte der eingeblendeten Präsentation zeigen (Abb. 2). Dafür ist es erforderlich, auf dem Kontrollbildschirm den Vortragenden ihr eigenes Spiegelbild zu zeigen (Abb. 5). Da aber alle Inhalte (Texte etc.) seitenrichtig sein sollen, ist es erforderlich, den Sprecher oder die Sprecherin im Video zu spiegeln. Für die Zuschauer erscheint somit auch im endgültigen Video ein Spiegelbild der vortragenden Person. Bei der gewählten Anordnung sehen die Zuschauer die Präsentierenden mit der "rechten" Hand auf etwas zeigen, tatsächlich muss aber die linke verwendet werden, was für Rechtshänder etwas Übung erfordert. Besonders vertrackt sind mit den Händen angedeutete Drehbewegungen oder die „Rechte-HandRegel“, da diese in entgegengesetzte Richtung vorgeführt werden müssen.

Die gesamte Technik wird sowohl für die Aufzeichnung von asynchron bereitgestellten Lehrvideos als auch zur Produktion von Livestreams verwendet. Die Software OBS bietet hierzu eine Schnittstelle für YouTube an.

Nach der Aufzeichnung der asynchronen Inhalte werden diese einer einfachen Nachbearbeitung mit der Open Source Software ShotCut unterzogen, bei der das Rohmaterial zusammengeschnitten und ggf. grobe Fehler beseitigt wurden. Die Produktion kann somit durch "live on tape" beschrieben werden.

Da das Studio in den Räumen der Professur aufgebaut ist, ist es einfacher möglich, Demonstrationen und Experimente aus dem Fundus in ein Video einzubinden, als entsprechendes Material in einen Hörsaal zu transportieren (Abb. 6). Davon soll in Zukunft noch intensiver Gebrauch gemacht werden.

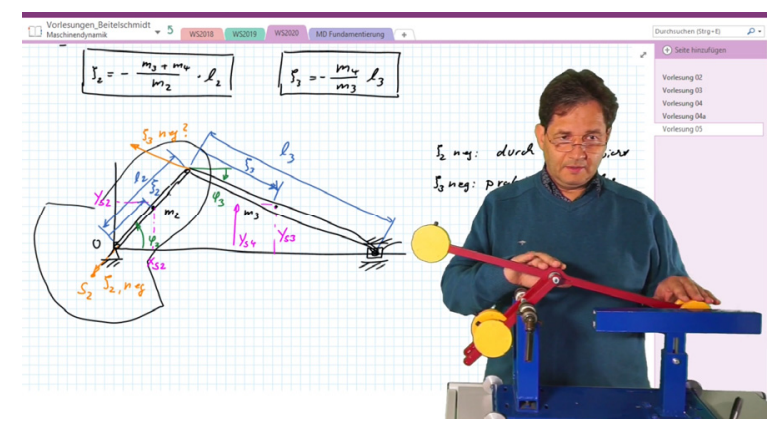

Abb. 6: Vorführung eines Experiments im Vorlesungs-Video

Folgende Veranstaltungen wurden im Wintersemester im Studio produziert:

1. Maschinendynamik

- $20 \mathrm{~h}$ Vorlesung

- $10 \mathrm{~h}$ Zentralübung + Übungseinführungen

2. Gekoppelte Simulation/Echtzeitsimulation (Englisch)

- $14 \mathrm{~h}$ YouTube Livestreams

3. MKS in der Fahrzeugtechnik

-10 h Vorlesung + Übungseinführungen

4. Einführungen in Online-Praktikumsversuche 
Insgesamt bewerten die Autoren und andere Mitarbeiter der Professur für Dynamik und Mechanismentechnik die Anwendung der Studio-Technik als großen Fortschritt, mit dem im Wintersemester 2021 sehr ansehnliche Videos produziert werden konnten. Auch die positiven Rückmeldungen der Studierenden, sowohl Einzelaussagen als auch die Lehrevaluation, bestätigen diese Ansicht.

Im Sommersemester 2021 wird das Studio weiter im Einsatz sein.

\section{Danksagung}

Wir danken der Fakultät Maschinenwesen für den Kauf der Kamera sowie dem ZIH der TU Dresden für die Ausleihe des Mikrofons. 